\title{
ALCOHOLIC LIVER DISEASE
}

\section{Insights into the pathogenesis of alcoholic liver disease}

The pathogenesis of alcoholic liver disease (ALD) is poorly understood. Improved understanding of this disease is urgently needed as two-thirds of patients with severe ALD die within 48 months of diagnosis. "However, the link between alcohol consumption and liver damage is not direct, as only one in six heavy drinkers go on to develop severe liver disease," says Yun Ma. Various mechanisms might be involved, including genetic susceptibility, direct ethanol toxicity and immune responses.

Patients with ALD have autoantibodies directed against alcohol dehydrogenase (ADH), which is a key enzyme involved in the metabolism of alcohol. In a study published in Hepatology, $\mathrm{Ma}$ and colleagues decided to extend their previous preliminary investigations exploring cellular immunity as a contributing factor in ALD. They aimed to characterize $\mathrm{ADH}$-specific T-cell responses in 39 patients with alcohol-related cirrhosis (12 actively drinking or abstinent for $<6$ months, 13 abstinent for $>6$ months and 14 undergoing liver transplantation).

The researchers used three immunological approaches in their investigation: first, they assessed

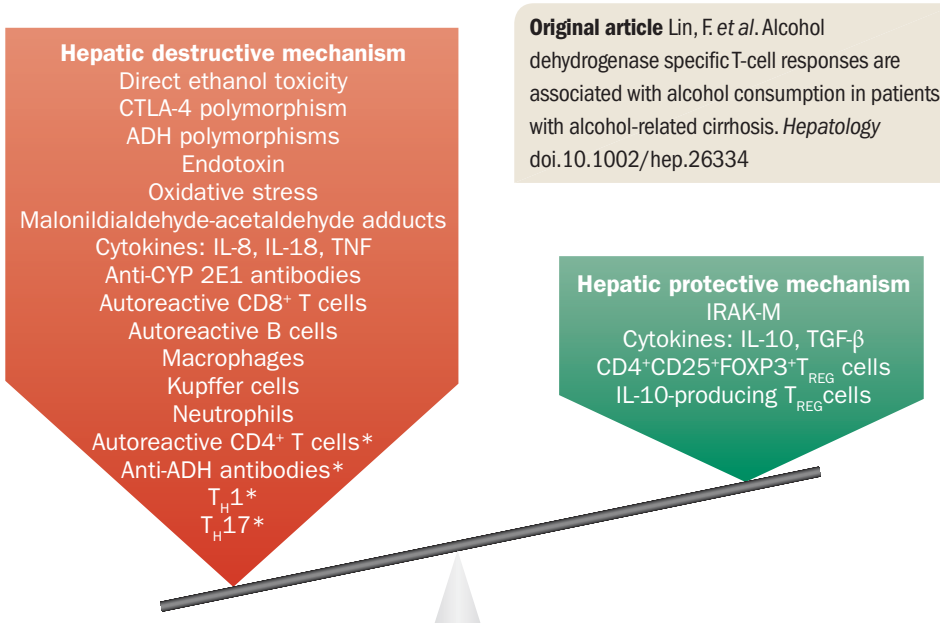

Possible mechanisms of alcoholic liver disease. *These mechanisms are being investigated by Ma and co-workers. Abbreviations: ADH, alcohol dehydrogenase; CTLA-4, cytotoxic T-lymphocyte antigen 4; TGF- $\beta$, transforming growth factor $\beta ; T_{H}$, T-helper cell; $T_{R E G}$, T-regulatory cell. Courtesy of $Y$. Ma.
T-cell proliferative responses after ADH peptide stimulation; second, they used flow cytometry to define the phenotypes of cytokineproducing T cells; and third, they used ELISA to measure the levels of cytokines released by $\mathrm{T}$ cells.

The main findings from the study were that immune responses were increased in the group of patients who were actively drinking or abstinent $<6$ months. "These immune responses involved T-helper type $1\left(\mathrm{~T}_{\mathrm{H}} 1\right)$ and $\mathrm{T}_{\mathrm{H}} 17$ cells, which cause liver damage," report $\mathrm{Ma}$ and co-workers. "In addition, there was an imbalance between $\mathrm{T}_{\mathrm{H}} 1$ and $\mathrm{T}_{\mathrm{H}} 2$ immune responses, which favours fibrogenesis." Interestingly, immune responses, although weaker, persisted in the abstinent patients.

"We plan to expand the current investigation to include a cohort of patients with alcoholic hepatitis; we will explore the role of adaptive immunity in the pathogenesis of this devastating condition," says Ma. "Furthermore, we will elucidate the mechanisms leading to $\mathrm{T}_{\mathrm{H}} 1 /$ $\mathrm{T}_{\mathrm{H}} 2$ imbalance." The researchers are hopeful that their findings will lead to improvements in treatments for patients with ALD, reducing the demand for liver transplantation.

\section{Isobel Leake}

dehydrogenase specific T-cell responses are with alcohol-related cirrhosis. Hepatolog oi.10.1002/hep.26334 